DOI: 10.30972/eitt.704770

\title{
Hábitos alimenticios de los jóvenes en tiempos de aislamiento social preventivo y obligatorio
}

\author{
Melisa J. Hidalgo'* ${ }^{*}$, Diana C. Fechner ${ }^{1}$, Ramón A. Martínez ${ }^{1}$, César A. \\ Lezcano $^{1}$, Eva G. S. Miño ${ }^{2}$, Lorena Obregónn ${ }^{3}$, Roberto G. Pellerano ${ }^{1}$
}

\section{Resumen}

En este trabajo se presenta los resultados de un estudio observacional realizado a través de una encuesta online sobre los hábitos alimenticios de jóvenes en edad escolar durante el aislamiento social preventivo y obligatorio debido a la pandemia de coronavirus. Los jóvenes encuestados pertenecen a dos colegios secundarios de la provincia de Corrientes. La información obtenida de las encuestas ha permitido estudiar el perfil de nutrición de dichas poblaciones bajo estudio a fin de convertirse en instrumentos para la planificación de futuros talleres y capacitaciones en relación a la educación alimentaria.

Palabras clave: alimentación; escuela; salud

\section{Introducción}

Con el objetivo de proteger la salud pública frente a la propagación del coronavirus se ha establecido el aislamiento social preventivo y obligatorio (ASPO) como una

\footnotetext{
(1) Departamento de quimica, Facultad de Ciencias Exactas y Naturales y Agrimensura, UNNE, Avenida Libertad 5470 , Corrientes, Argentina.

(2) Colegio Secundario "Dr. Luis Federico Leloir", Avenida La Paz 3400, Corrientes, Argentina.

(3) Colegio Secundario Colonia Llano, Ruta Provincial N4, San Luis del Palmar, Corrientes, Argentina

*E-mail: roberto.pellerano@comunidad.unne.edu.ar (Director)
} 
medida excepcional en todo el territorio argentino. La enfermedad provocada por el coronavirus es una enfermedad de tipo infecciosa capaz de transmitirse de persona a persona, es causada por el virus SARS-CoV-2 descubierto recientemente y que ha causado una pandemia a nivel global amenazando la seguridad mundial [1].

Esta medida de aislamiento ha llevado a que las personas temporalmente deban permanecer en sus domicilios permitiendo el mínimo desplazamiento para proveerse de alimentos, artículos de limpieza y medicamentos. Para de esta manera reducir el contacto y minimizar las posibilidades de contagio. Esta situación, indudablemente, afectó el estilo de vida de la comunidad en general incluido la forma en que esta se alimenta.

En este sentido, nos enfocamos en realizar una aproximación a la nutrición de los jóvenes durante el ASPO. Partiendo de la base que mantener una buena nutrición, una dieta suficiente y equilibrada, en combinación con la práctica de ejercicio físico regularmente, constituye uno de los pilares para tener una buena salud [2].

Nuestra investigación se centra en los jóvenes en edad escolar debido a que en esta etapa de la vida acontecen cambios importantes desde diferentes aspectos como ser a nivel físico, hormonal, social y psicoemocional siendo fundamental que la alimentación permita un adecuado desarrollo y crecimiento. Es en este periodo que los individuos presentan una alta velocidad de crecimiento corporal, elevando los requerimientos energéticos y nutricionales, variando de un sexo a otro. Adicionalmente, los jóvenes adquieren por lo general nuevos hábitos de consumo de alimentos, en relación a influencias del entorno social, los cambios en el estilo de vida y la búsqueda de autonomía. Por este motivo se deben promover hábitos de vida saludables para prevenir trastornos nutricionales y prepararlos nutricionalmente para tener una vida adulta más sana [3].

Por todas estas razones, los integrantes del proyecto titulado "Minerales esenciales y su suplementación” del programa la Universidad en el Medio (UNNE) llevamos a cabo la realización de un análisis exploratorio sobre los hábitos alimentarios de estudiantes jóvenes que asisten a dos colegios secundarios de la provincia de Corrientes. La recolección de los datos se llevó a cabo a través de una encuesta tipo 
online, realizada en la población de alumnos de dos colegios con contextos distintos, uno ubicado en la capital de la Provincia de Corrientes (Colegio secundario Dr. L.F. Leloir) y el otro en un contexto rural del departamento de San Luis del Palmar (Colegio secundario Colonia Llano, a $22 \mathrm{~km}$ de la capital correntina). Las respuestas de los alumnos encuestados fueron analizadas y contrastadas con guías alimentarias como así también artículos científicos relacionados con la temática. Las referencias utilizadas sirvieron como criterio para establecer frecuencias y cantidades a consumir de los grandes grupos alimenticios para la franja etaria de las poblaciones bajo estudio.

\section{Metodología}

Los integrantes del proyecto UNNE en el medio, "Minerales esenciales y su suplementación”, a través de sucesivas reuniones virtuales llevaron adelante la confección de una encuesta virtual conformada por nueve preguntas (ANEXO I) para que los alumnos encuestados respondan sobre los diferentes grupos de alimentos y cambios de hábitos alimentarios durante el ASPO. Para la realización de las encuestas virtuales se contó con la colaboración de los docentes y autoridades de los establecimientos que también forman parte del proyecto. Los colegios en los que se realizaron las encuestas fueron:

- Colegio secundario Dr. Luis Federico Leloir ubicado en avenida La Paz al 3400 en el barrio Cacique Canindeyú de la ciudad de Corrientes. La matrícula del colegio es de aproximadamente 1.700 alumnos por año, consta de un ciclo básico $\left(1^{\circ}, 2^{\circ}\right.$ y $3^{\circ}$ año) y un ciclo orientado $\left(4^{\circ}, 5^{\circ}\right.$ y $6^{\circ}$ año), en el ciclo orientado existen las modalidades de bachiller en Ciencias Sociales; Ciencias Naturales; Economía y Administración. Además, el colegio cuenta con una huerta escolar. Los alumnos que participaron de la encuesta pertenecen a los distintos ciclos, en donde desarrollan la asignatura química. En total son 226 alumnos, en edades comprendidas entre los 13 a 18 años, los cuales recibieron y respondieron la encuesta a través de un formulario de Google.

- Colegio Secundario Colonia Llano se encuentra ubicado en la localidad de San Luis del Palmar-Primera Sección a 7 kilómetros de la zona céntrica del pueblo. Se accede al mismo por camino enripiado (Ruta 4) y los alumnos por lo general 
se transportan en vehículos particulares (motos), minibús y colectivo escolar. El colegio cuenta con una matrícula de aproximadamente 250 alumnos distribuidos en ciclo básico y ciclo orientado. Cuenta actualmente con dos divisiones en el ciclo orientado, una con formación en agroambiental y otra con orientación en educación física. La población escolar es variada ya que cuenta con alumnos del pueblo vecino (San Luis del Palmar de aproximadamente 12.00o habitantes) pero también de la zona rural (paraje Colonia Llano y aledaños), alumnos repitentes y con sobreedad escolar, también muchos tienen otras obligaciones como el trabajo y responsabilidades de familia propia. Los alumnos que participaron de la encuesta pertenecen al ciclo orientado, específicamente a $5^{\circ}$ y $6^{\circ}$ en ambos cursos desarrollan la asignatura química. En total son 30 alumnos de los cuales solo algunos (n = 12 alumnos) pudieron responder a la encuesta debido a la realidad actual, educación a distancia a causa de la pandemia. Recibieron y respondieron la encuesta a través del WhatsApp, única vía de comunicación con la que actualmente cuentan los docentes para el envío y recepción de trabajos prácticos y evaluaciones, además de las consultas y explicaciones que se envían periódicamente para el desarrollo de los mismos. Los resultados obtenidos se presentan y discuten en la siguiente sección.

\section{Resultados y Discusión}

El procedimiento de encuesta online tuvo como fin recopilar información sobre las dos poblaciones bajo estudio, formando parte de una de las primeras experiencias del proyecto de extensión. En relación con los resultados obtenidos, en primera instancia, debemos destacar que aproximadamente el 64\% (144 individuos) de los alumnos encuestados del Colegio Leloir se encuentra en el rango etario de 13-15 años mientras que el 36\% restante (82 individuos) pertenece a la franja etaria de 16 a 18 años. Los alumnos encuestados del Colegio Colonia Llano correspondieron al rango etario de 16 a 21 años, esto debido a que como se indicó en la sección Metodología algunos estudiantes del Colegio Colonia Llano presentan sobreedad escolar. En cuanto al género de los encuestados, en el Colegio Leloir 141 estudiantes (62,2\%) corresponden al género femenino y $85(37,8 \%)$ al género masculino, mientras que en el Colegio Colonia Llano se observaron porcentajes en el mismo orden $58 \%$ femenino y $42 \%$ masculino. 
En cuanto a las respuestas de los alumnos, la primera pregunta de la encuesta sobre los grupos de alimentos se refiere al consumo de frutas. Esto debido a la importancia que tiene el consumo diario de frutas y verduras que se encuentra relacionado con una disminución en el riesgo de padecer ciertas patologías como ser diabetes, obesidad, cáncer de colon y enfermedades cardiovasculares. Las respuestas obtenidas mostraron que la mayoría de los alumnos del colegio urbano respondieron que consumen entre 1 y 2 frutas por día (55,6\%), mientras que un 23,6\% de los jóvenes encuestados declaró consumir 3 o más frutas. Resulta preocupante que aproximadamente un $21 \%$ de los alumnos declaró no consumir frutas.

Respecto a los alumnos del colegio rural, el 25\% respondió consumir entre 1 y 2 frutas por día, 42\%, 3 o más. Con respecto a la proporción de alumnos que declara no consumir frutas el porcentaje fue mayor (33\%) que, en el colegio urbano, esto posiblemente debido a los rangos etarios de las poblaciones encuestadas. En la Figura 1 se puede observar con mayor detalle las respuestas brindadas por los alumnos de ambos colegios.

En este contexto resulta importante destacar que el Ministerio de Salud de Argentina recomienda consumir dos o más frutas diarias [4], con lo que se demuestra que la mayoría de la población estudiada en ambos colegios cumple con este requisito (aproximadamente $57 \%$ de los encuestados en el colegio urbano y 50\% de los encuestados en el colegio rural).

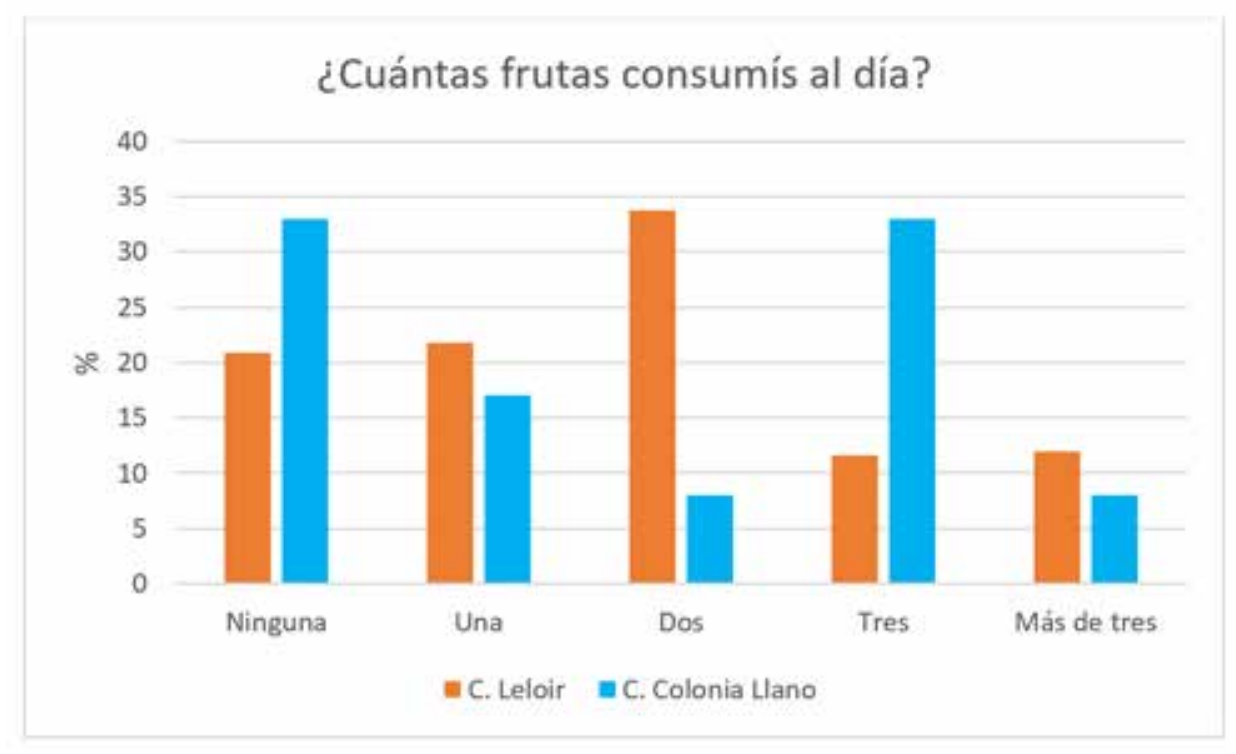

Figura 1. Resultados obtenidos en porcentajes sobre el consumo de frutas en los dos colegios. 
La segunda pregunta de la encuesta se refirió al consumo de productos lácteos (Figura 2). Se observó que la mayoría de los alumnos del colegio urbano refirieron consumir al menos uno o dos productos lácteos por día (55\%), mientras que el $42 \%$ de los encuestados del colegio rural afirmó consumir esta cantidad diaria de productos lácteos. Los alumnos que afirmaron consumir tres o más de tres productos lácteos por día en el colegio urbano alcanzaron niveles similares a los encuestados del colegio rural un 25\% aproximadamente. Con respecto a los alumnos que afirman no consumir productos lácteos en el colegio urbano fueron la minoría alcanzando aproximadamente un $8 \%$ mientras que en el colegio rural una fracción importante de los alumnos afirmó no consumir productos lácteos (33\%). Este último dato resulta de importancia sobre la necesidad de generar conciencia respecto a los hábitos alimenticios, téngase en cuenta que según recomendaciones del Ministerio de Salud se debe incluir al menos tres porciones al día de leche, yogurt o queso, dada la importancia de este grupo de alimentos para mantener niveles adecuados de ingesta de calcio [4]. Este nutriente mineral es esencial a largo de la vida, especialmente en la adolescencia donde aproximadamente el 40\% de la formación ósea ocurre en este periodo de la vida. El requerimiento diario de este nutriente se encuentra entre 1200 y $1500 \mathrm{mg} /$ día [5].

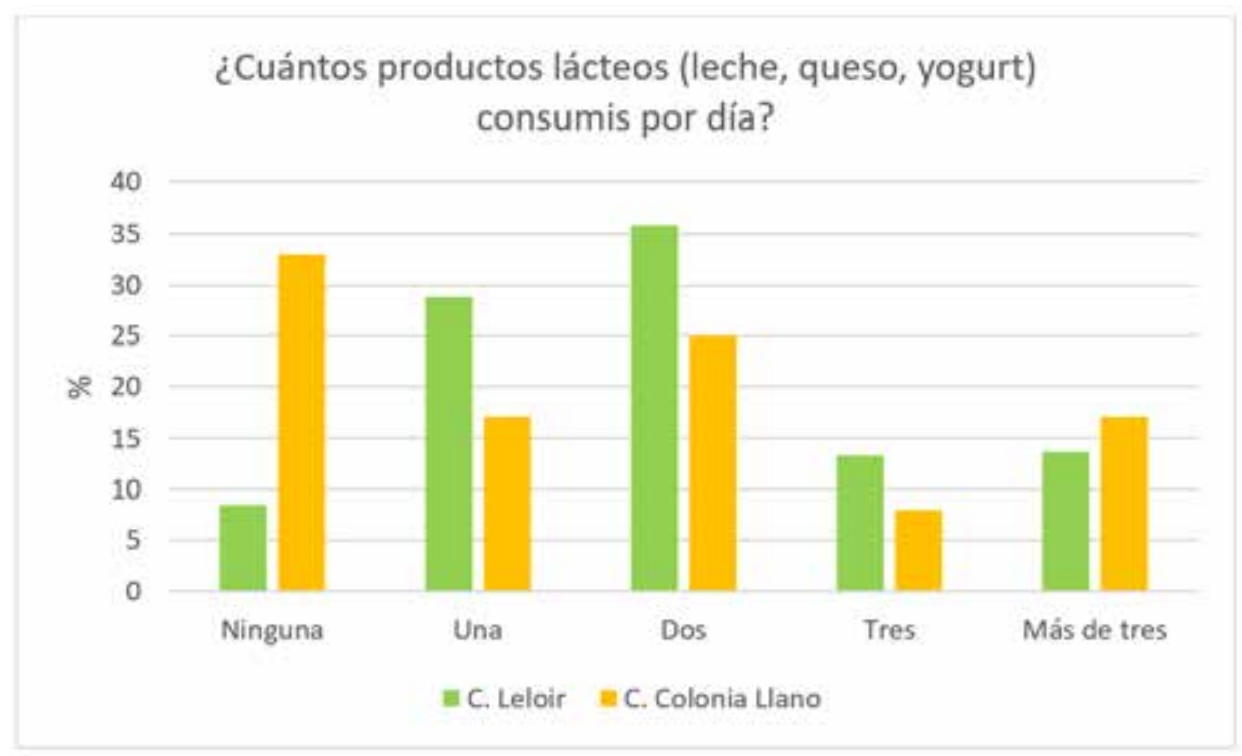

Figura 2. Resultados obtenidos en porcentajes sobre el consumo de productos lácteos en los dos colegios.

La tercera pregunta de la encuesta se refiere al consumo de carne diario de los alumnos. El consumo aparente de proteínas de la población argentina se mantuvo en un rango de 90,1 a 108,2 g/hab/día desde el año 1961 a 2015 [4]. Pero hay que 
tener en cuenta que el adolescente tiene necesidades nutricionales aumentadas debidas a la velocidad de crecimiento. Estas necesidades dependen de factores genéticos, constitucionales y del sexo, entre otros.

En cuanto a las proteínas, la cantidad recomendada es de $0,95 \mathrm{~g} / \mathrm{kg} /$ día para el grupo de 10 a 13 años, y de $0,85 \mathrm{~g} / \mathrm{kg} /$ día para el grupo de 14 a 18 años, lo que significa un aporte aproximado de $34 \mathrm{~g} /$ día de 9 a 13 años, y de $52 \mathrm{~g} /$ día en varones y $46 \mathrm{~g} /$ día en mujeres para el grupo de mayor edad. Esto sería, en un plan alimentario promedio, 130 gramos de carne por día, que junto al consumo de huevo, queso y leche/yogur aportarían las proteínas diarias necesarias. Por otra parte, hay que tener en cuenta que las proteínas que aportan los alimentos de origen animal (leche, quesos, carnes, huevos) se aprovechan mejor que las de los alimentos de origen vegetal (cereales, legumbres, hortalizas) porque estas últimas son más incompletas.

Teniendo en cuenta los resultados de la encuesta, se puede afirmar que ambas poblaciones encuestadas manifestaron mayoritariamente consumir carne a diario, aproximadamente $49 \%$ de los alumnos encuestados de la escuela urbana y $75 \%$ de los alumnos del colegio rural, es decir que satisfacen los requerimientos diarios de proteínas, ya que ingieren carne todos los días (Figura 3). Respecto de los estudiantes que consumen carne solamente una o dos veces por semana en ambos colegios resultaron minoritarios, aproximadamente un $20 \%$ en el colegio urbano y un $8 \%$ en

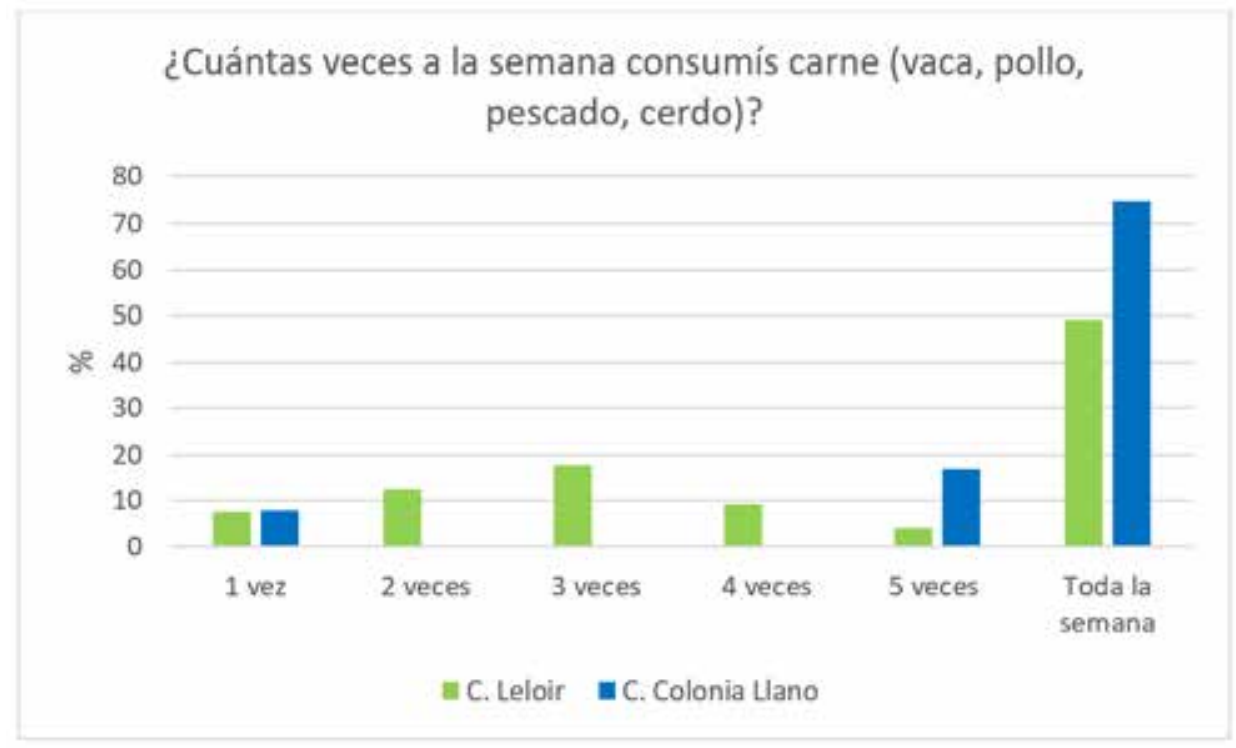

Figura 3. Resultados obtenidos en porcentajes sobre el consumo de carne en los dos colegios. 
el colegio rural. Con estos resultados, no es posible afirmar que los alumnos poseen una ingesta deficiente de proteínas, debido a que no se correlaciona la información de la ingesta de carne con la de otras fuentes de proteína (huevos, lácteos, legumbre/cereales). Es recomendable entonces que el grupo de menor consumo de carne tenga una ingesta mayor de otras fuentes de proteínas los días que no ingieren carne, para alcanzar la dosis diaria de proteína.

La cuarta pregunta de la encuesta se refiere al consumo de comida elaborada o comida rápida. El consumo de comidas rápidas es cada vez más frecuente, aunque no solamente entre la población juvenil. Así, están alcanzando gran popularidad las comidas precocinadas para consumir incluso en casa. Las comidas rápidas tendrían casi un 50\% de calorías procedentes de las grasas, aunque actualmente este porcentaje ha disminuido. Además, este tipo de comidas tienen un exceso de proteínas y sal. La repercusión nutricional en el adolescente depende de la frecuencia de su consumo y de los alimentos que forman el resto de la dieta diaria. La actitud frente al consumo de comidas rápidas, que además son atractivas para el joven, debe ser flexible, pues forma parte de la evolución propia del mundo occidental. La recomendación de las guías alimentarias es limitar el número de comidas rápidas, intentar que en este tipo de comidas se incluyan las ensaladas y compensar los posibles desequilibrios de los distintos nutrientes con las comidas que hacen en su propia casa.

En la encuesta se preguntó cuántas veces por semana consumen comida comprada, lo cual no necesariamente es lo conocido como “comida rápida”. A veces pueden ser viandas o comidas saludables, pero para ello es importante conocer la forma de elaboración y la materia prima empleada en su elaboración. Del total de encuestados del colegio urbano aproximadamente un 4\% de los alumnos manifestó consumir comida comprada 4 veces por semana mientras que en el colegio rural el 17\% de los alumnos manifestaron este nivel de consumo. Esta respuesta resulta esperable debido a la disponibilidad de lugares donde acceder a este tipo de alimentos. En ambos colegios la mayoría de los encuestados manifestaron consumir comida comprada al menos una o dos veces por semana (Figura 4). Generalmente la comida comprada a la que se refirieron los alumnos que desearon hacerlo se refirieron mayoritariamente a alimentos fritos, lo cual según las recomendaciones del Minis- 
terio de Salud es importante no abusar de ellos y compensar la nutrición con otros alimentos que sean saludables.

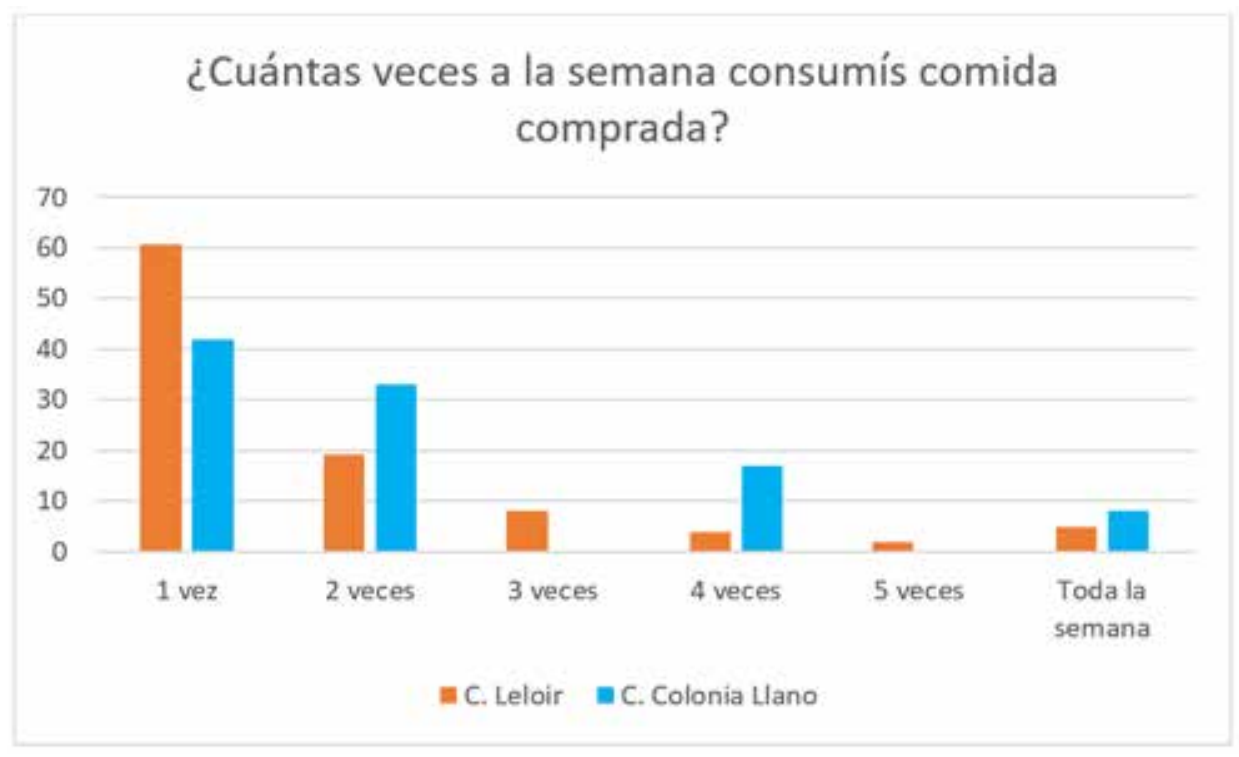

Figura 4. Resultados obtenidos en porcentajes sobre el consumo de comida comprada en los dos colegios.

La quinta pregunta realizada en la encuesta busca indagar sobre la correcta hidratación y tipos de líquidos que consumen. Una correcta hidratación es muy importante para garantizar un adecuado balance hídrico en el cuerpo, entre el líquido que se bebe y el que se pierde; y favorecer así a un óptimo rendimiento mental y físico. Los requerimientos de agua varían entre personas y según las diferentes condiciones ambientales. Por eso varían las cantidades recomendadas, pero en la Argentina se acordó, junto a un panel de expertos vinculados a la nutrición y el metabolismo, una recomendación de ingesta de líquidos (preferentemente agua) de 2 a 2,5 l/día/ persona, no estableciéndose un límite máximo de su ingesta. Estos requerimientos se incrementan cuando las temperaturas son altas y durante la actividad física, ya que se pierde más liquido por la sudoración.

Del análisis de los resultados obtenidos se infiere que el $47 \%$ los adolescentes encuestados en la escuela urbana consumen menos de la cantidad diaria recomendada de líquido, la cual es de 2,5 litros para adolescentes masculinos y de 2 litros para adolescentes femeninas. A su vez, similares porcentajes se observan de la encuesta realizada en la escuela rural. La posible razón del bajo consumo de líquido 
puede deberse: 1) condiciones climáticas y temperatura ambiental al momento de realizarse la encuesta; 2) baja actividad física debido a la situación de pandemia y el aislamiento social, preventivo y obligatoria; 3) poca información respecto de la importancia de la hidratación.

Con respecto a los alumnos que consumen cantidades recomendadas, es de resaltar que 29\% consume más de 2 litros para los alumnos del colegio urbano, y una proporción similar (25\%) para los alumnos de la escuela rural.

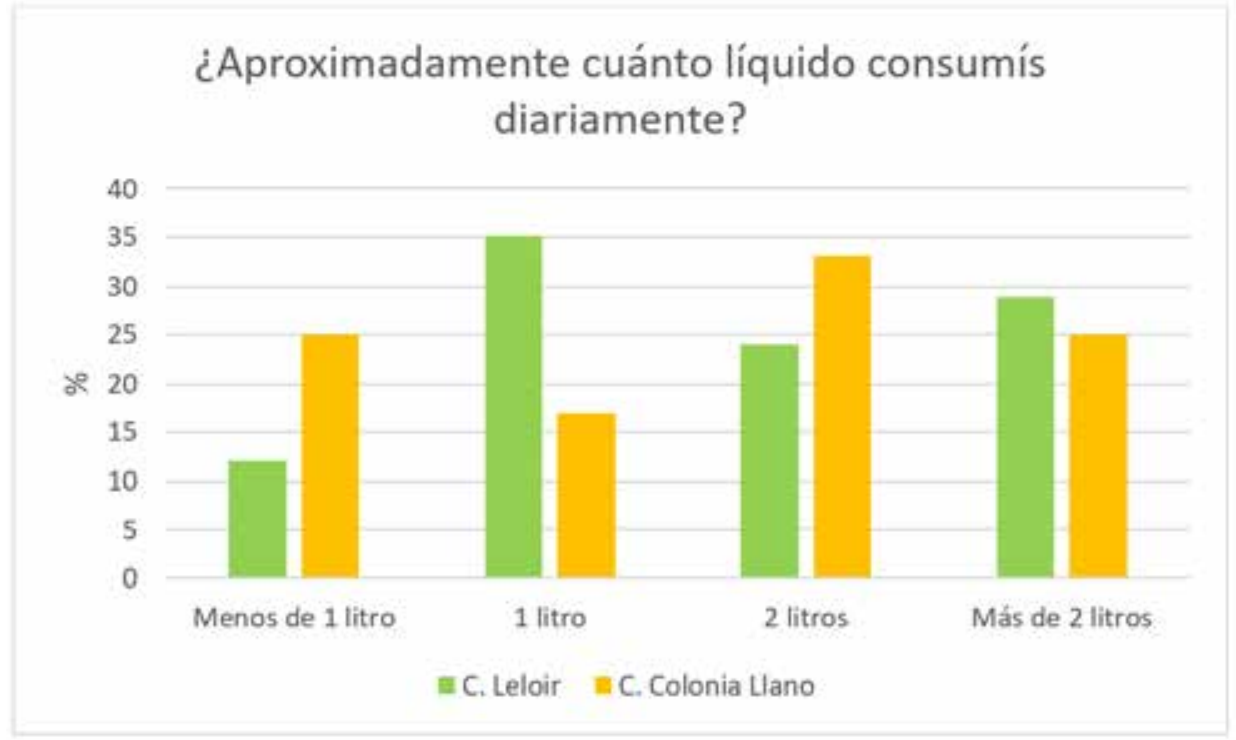

Figura 5. Resultados obtenidos en porcentajes sobre el consumo aproximado de líquido en los dos colegios.

Respecto al tipo debida utilizada para hidratarse, la encuesta incluyó una pregunta para que los alumnos identifiquen la fuente de hidratación utilizada (Figura 6). Una de las bebidas más consumidas por la población encuestada de ambos colegios resultó ser el agua, estos resultados resultaron superiores a estudios publicados en la bibliografía actual, donde se indicaban valores del 22\% de agua en la ingesta total de líquidos diarios [6,7]. A continuación, entre las bebidas preferidas de la población encuestada aparecen las infusiones de yerba mate (mate/tereré). Este es un dato alentador (siempre y cuando las infusiones no sean azucaradas o endulzadas), ya que evidencia un bajo consumo de bebidas artificiales por parte de la población adolescente. En tercer y cuarto lugar de consumo se encuentran las gaseosas/agua saborizadas y café/té. 


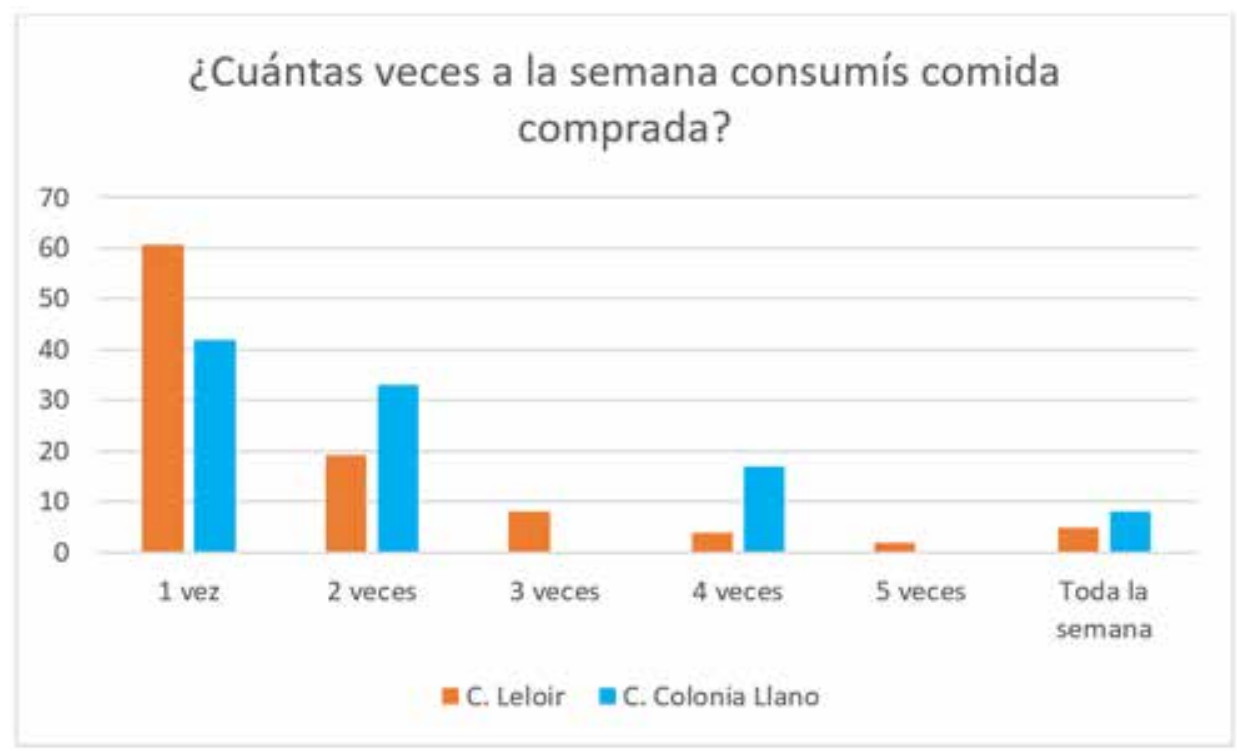

Figura 6. Resultados obtenidos en porcentajes sobre cuáles son los líquidos que mayormente consumen en los dos colegios

Finalmente, referente a los cambios de costumbres alimenticias en este tiempo de aislamiento, la mayoría de los alumnos de ambos colegios contestaron que si se produjeron cambios en sus hábitos. La bibliografía al respecto hace referencia a que los cambios de los hábitos alimenticios pueden deberse al aumento de ansiedad, sedentarismo y estrés a causa del aislamiento, como así también las dificultades de acceso a los puntos de venta de alimentos entre otros factores [8].

\section{Conclusiones}

En conclusión, a través de la encuesta realizada de manera virtual a alumnos que asisten a diferentes colegios secundarios de la provincia de Corrientes, por un lado, un establecimiento ubicado en la ciudad de Corrientes y por el otro un establecimiento situado en un sector rural correspondiente a la localidad de San Luis del Palmar (a $22 \mathrm{~km}$ de la ciudad) nos ha permitido realizar un relevamiento respecto a la ingesta de alimentos de los jóvenes durante esta etapa de ASPO provocado por la enfermedad infecciosa de coronavirus 2019. En este sentido debemos destacar la buena predisposición e interés para la realización de la encuesta tanto de los alumnos como los docentes y autoridades de los colegios secundarios. La información obtenida servirá de base para diseñar los materiales de difusión en relación a una nutrición saludable y hábitos alimenticios para esta etapa de desarrollo del ser humano en estas dos poblaciones bajo estudio. 


\section{BIBLIOGRAFIA}

[1] Ministerio de Salud. (2020). Aislamiento social, preventivo y obligatorio. Disponible en: https://www.argentina.gob.ar/coronavirus/aislamiento

[2] Organización Mundial de la Salud, OMS. (2020). Nutrición. Disponible en: https://www.who.int/topics/nutrition/es/

[3] Marugán de Miguelsanz, J. M., Monasterio Corral, L., \& Pavón Belinchón, M. P. (2010). Alimentación en el adolescente. H. y Asociación Española de Pediatría-Sociedad Española de Gastroenterología, Protocolos diagnóstico-terapéuticos de Gastroenterología, Hepatología y Nutrición Pediátrica. Santiago de Compostola: ERGON.

[4] Ministerio de Salud. (2016). Guías alimentarias para la población Argentina. Disponible en: https://nutricion.fcm.unc.edu.ar/wp-content/uploads/sites/16/2010/11/Guia_Alimentaria_completa.pdf

[5] Fernandez, A., Sosa, D. P., Setton, D. D., Virginia, D., Fabeiro, D. M., Maria, D., \& Hernandez, D. J. (2011). Calcio y nutrición. Sociedad Argentina de Pediatría, 1-19.

[6] Asociación Argentina de Dietistas y Nutricionistas Dietistas. (2016). Gacetilla de prensa "Hidratación Saludable”. Buenos Aires, Argentina.

[7] Gupta, N., Goel, K., Shah, P., \& Misra, A. (2012). Childhood obesity in developing countries: epidemiology, determinants, and prevention. Endocrine reviews, 33(1), 48-70.

[8] Rodríguez Osiac, L. (2020). Evitemos la inseguridad alimentaria en tiempos de COVID-19 en Chile. Revista chilena de nutrición, 47(3), 347-349.

\section{ANEXO I | Modelo de la encuesta}

Proyecto “Minerales Esenciales y su Suplementación” del programa la Universidad en el Medio (FaCENA - UNNE)

La siguiente encuesta es para conocer los hábitos alimentarios en estos tiempos de aislamiento social preventivo y obligatorio.

\section{1. ¿Cuántos años tienes?}

a. Entre 13 a 15 años

b. Entre 16 a 18 años 
2. Selecciona tu género:

a. Femenino

b. Masculino

3. ¿Cuántas frutas consumís al día?

a. Ninguna

b. Una

c. Dos

d. Tres

e. Más de tres

4. ¿Cuántas porciones de productos lácteos (leche, queso, yogurt) consumís por día?

a. Ninguna

b. Una

c. Dos

d. Tres

e. Más de tres

5. ¿Cuántas veces a la semana consumís carne (vaca/pollo/cerdo/ pescado)?
a. 1 vez
b. 2 veces
c. 3 veces
d. 4 veces
e. 5 veces
f. Toda la semana
6. ¿Cuántas veces a la semana consumís comida comprada?
a. $1 \mathrm{vez}$
b. 2 veces
c. 3 veces
d. 4 veces
e. 5 veces
f. Todos los días
7. ¿Aproximadamente cuánto líquido consumís diariamente?
a. menos de 1 litro
b. 1 litro 
c. 2 litros

d. Más de 2 litros

8. ¿Cuáles son los líquidos que mayormente consumís? Puede ser más de una opción.
a. agua
b. gaseosa / agua saborizada
c. mate / tereré
d. café / té

9. ¿Consideras que cambiaste tus costumbres alimenticias en este tiempo de aislamiento?
a. $\mathrm{Si}$
b. No 